BULL. AUSTRAL. MATH. SOC.

VOL. 25 (1982), 433-440.

\title{
GENERATORS OF SIMPLE ALGEBRAS
}

\author{
David Choate
}

\begin{abstract}
It is shown that simple algebras of characteristic not equal to 2 , which contain non-trivial elements that satisfy $x^{n}=x$ for some fixed, minimal integer $n>1$, are generated by these elements.
\end{abstract}

\section{Introduction}

In the study of simple rings idempotents play an important role. For example, it is known that simple algebras which contain non-trivial idempotents are generated by their idempotents (with some exceptions in characteristic two). We will generalize this result by relaxing the hypothesis of a theorem of Jacobson which says that if $R$ is a ring in which $a^{n}=a$ (with $n>1$ an integer depending on $a$ ) for every $a \in R$ then $R$ must be commutative. We require only that there exists a nontrivial element $a$ such that $a^{n}=a$ for some integer $n>1$. With this we can show that some simple algebras at least are "more nearly commutative" in the sense that the commutator $[R, R]$ is contained in a subset of a set which is known to contain it.

\section{Definitions}

We call an element $a$ an n-potent provided $a^{n}=a$ but $a^{t} \neq a$ for $1<t<n$. An $n$-potent is non-trivial provided $a^{n-1} \neq 1,0$. Observe that $a^{n-1}$ is a non-trivial idempotent since

Received 6 January 1982. 


$$
\left(a^{n-1}\right)^{2}=a^{2 n-2}=a^{n} a^{n-2}=a^{n-1}
$$

By $E_{n}^{n-1}$ we mean the additive group generated by the $(n-1)$ st powers of non-trivial $n$-potents. It is clear from the above that $E_{n}^{n-1} \subset E^{\prime}=E_{2}^{l}$.

\section{The Lie ideal $E_{n}^{n-1}$}

LEMMA 1. If a is a non-trivial n-potent in a ring $R$, then so are $a+a^{n-1} r-a^{n-1} r a^{n-1}$ and $a+r a^{n-1}-a^{n-1} r a^{n-1}$ for every $r \in R$.

Proof. We first show that

$$
\left(a+a^{n-1} r-a^{n-1} r a^{n-1}\right)^{k}=a^{k-1}\left(a+a^{n-1} r-a^{n-1} r a^{n-1}\right)
$$

by induction. This equation is clearly true for $k=1$. Assume it is true for some $k \geq 1$. Then

$$
\begin{aligned}
\left(a+a^{n-1} r-a^{n-1} r a^{n-1}\right)^{k+1} & \\
& =\left(a+a^{n-1} r \ldots a^{n-1} r a^{n-1}\right)^{k}\left(a+a^{n-1} r-a^{n-1} r a^{n-1}\right) \\
& =a^{k-1}\left(a+a^{n-1} r-a^{n-1} r a^{n-1}\right)\left(a+a^{n-1} r-a^{n-1} r a^{n-1}\right) \\
& =a^{k-1}\left(\left(a^{2}+a^{n} r-a_{r a}^{n-1}\right)+\left(a^{n-1} r a+a^{n-1} r a^{n-1} r-a^{n-1} r a^{n-1} r a^{n-1}\right)\right. \\
& \left.+\left(-a^{n-1} r a^{n}-a^{n-1} r a^{2 n-2} r+a^{n-1} r a^{2 n-2} r a^{n-1}\right)\right) \\
& =a^{k}\left(a+a^{n-1} r-a^{n-1} r a^{n-1}\right)
\end{aligned}
$$

since $a^{n-1}$ is an idempotent.

$$
\begin{aligned}
& \text { So if } k=n \text {, } \\
& \left(a+a^{n-1} r-a^{n-1} r a^{n-1}\right)^{n}=a^{n-1}\left(a+a^{n-1} r-a^{n-1} r a^{n-1}\right)=a+a^{n-1} r-a^{n-1} r a^{n-1} .
\end{aligned}
$$

Moreover if

$$
\left(a+a^{n-1} r-a^{n-1} r a^{n-1}\right)^{t}=a+a^{n-1} r-a^{n-1} r a^{n-1}
$$

for some $t, I<t<n$, we have

$$
a^{t-1}\left(a+a^{n-1} r-a^{n-1} r a^{n-1}\right)=a+a^{n-1} r-a^{n-1} r a^{n-1} \text {. }
$$

Multiplying from the right by $a^{n-1}$, we have $a^{t-1} a^{n}=a^{n}$, or $a^{t}=a$. But this is impossible since $a$ is an $n$-potent. So 
$a+a^{n-1} r-a^{n-1} r a^{n-1}$ is an $n$-potent.

Now if

$$
0=\left(a+a^{n-1} r-a^{n-1} r a^{n-1}\right)^{n-1}=a^{n-2}\left(a+a^{n-1} r-a^{n-1} r a^{n-1}\right),
$$

we can multiply from the right by $a^{n-1}$ to obtain

$$
0=a^{n-2} a^{n}=a^{n-2} a=a^{n-1} \text {. }
$$

But this is impossible since $a$ is non-trivial.

Also if

$$
1=\left(a+a^{n-1} r-a^{n-1} r a^{n-1}\right)^{n-1}=a^{n-2}\left(a+a^{n-1} r-a^{n-1} r a^{n-1}\right),
$$

we can multiply from the left by $a^{2}$ to get

$$
\begin{aligned}
a^{2} & =a^{n}\left(a+a^{n-1} r-a^{n-1} r a^{n-1}\right) \\
& =a\left(a+a^{n-1} r-a^{n-1} r a^{n-1}\right) \\
& =a^{2}+a^{n-1} r-a^{n-1} r a^{n-1} .
\end{aligned}
$$

Subtracting $a^{2}$ we find that $a^{n-1} r-a^{n-1} r a^{n-1}=0$. Substituting this in the first equation, we have $1=a^{n-1}$, a contradiction.

Therefore $a+a^{n-1} r-a^{n-1} r a^{n-1}$ is a non-trivial n-potent. In a similar manner we can show that $a+r a^{n-1}-a^{n-1} r a^{n-1}$ is also a nontrivial. $n$-potent.

THEOREM 1. Let $R$ be a ring with a non-trivial n-potent. Then $E_{n}^{n-1}$ is a Lie ideal of $R$.

Proof. Since $a$ is a non-trivial $n$-potent, so are $a+a^{n-1} r-a^{n-1} r a^{n-1}$ and $a+r a^{n-1}-a^{n-1} r a^{n-1}$ by Lemma 1. Therefore $\left(a+a^{n-1} r-a^{n-1} r a^{n-1}\right)^{n-1}=a^{n-2}\left(a+a^{n-1} r-a^{n-1} r a^{n-1}\right)=a^{n-1}+a^{n-2} r-a^{n-2} r a^{n-1}$ and

$$
\left(a+r a^{n-1}-a^{n-1} r a^{n-1}\right)^{n-1}=a^{n-1}+r a^{n-2}-a^{n-1} r a^{n-2}
$$


are elements of $E_{n}^{n-1}$ for each element $r \in R$. Replacing $r$ with ar in tne first and $r$ with $r a$ in the second, we find that $a^{n-1}+a^{n-1} r-a^{n-1} r a^{n-1}$ and $a^{n-1}+r a^{n-1}-a^{n-1} r a^{n-1}$ are elements of $E_{n}^{n-1}$. Since $E_{n}^{n-1}$ is an additive group, it contains $a^{n-1} r-r a^{n-1}=\left(a^{n-1}+a^{n-1} r-a^{n-1} r a^{n-1}\right)-\left(a^{n-1}+r a^{n-1}-a^{n-1} r a^{n-1}\right)$. Since $E_{n}^{n-1}$ is generated by the $a^{n-1}$ 's, $E_{n}^{n-1}$ is a tie ideal of $R$. COROLLARY 1.1. Let $A$ be a simple algebra of characteristic not equal to 2 having a non-trivial n-potent. Then $[A, A] \subset E_{n}^{n-1}$.

Proof. Since $E_{n}^{n-1}$ is a Lie ideal of $A$, we know by a celebrated theorem of Herstein [1, Theorem 1.5] that if $A$ is a simple algebra either $E_{n}^{n-1}$ is contained in the center of $A$ or $[A, A] \subset E_{n}^{n-1}$ unless $A$ is of characteristic 2 . The first possibility can be eliminated since the center of $A$ is a field and $E_{n}^{n-1}$ contains non-trivial idempotents.

COROLLARY 1.2. Let $A$ be a simple algebra of characteristic not equal to 2 having a non-trivial n-potent. Then the subring generated by its n-potents is $A$.

Proof. Let $S$ be the subring generated by the non-trivial $n$-potents. Then $S \supset E_{n}^{n-1} \supset[A, A]$ by Corollary 1.1. By a corollary to Herstein's theorem we know that the subring generated by $[A, A]$ is $A$. So $S$ contains $A$.

It is natural to ask if $E_{n}^{n-1}$ can be properly contained in $E$. The following example shows that this can happen when $n$ is 1 more than an odd prime.

EXAMPLE 1. Let $R$ be the set of $(p+1) \times(p+1)$ matrices over the rational numbers. We first show that $\frac{E_{p+1}^{p}}{2} \not$. If 


$$
\left(\begin{array}{ccccc}
0 & 1 & 0 & \ldots & 0 \\
0 & 0 & 1 & \ldots & 0 \\
\vdots & & & & \vdots \\
0 & 0 & 0 & \ldots & 1 \\
0 & +1 & 0 & \ldots & 0
\end{array}\right)_{(p+1) \times(p+1)}=a
$$

then $a$ is the companion matrix of the polynomial $f(x)=-x+x^{p+1}$. Since $f$ is the minimal polynomial for $a, a$ is a $(p+1)$-potent. If $a^{p}=1, a$ is invertible. But this is impossible since $a$ has a column of zeros. If $a^{p}=0, a=a^{p+1}=0$. So $a$ is a non-trivial $(p+1)-$ potent. This means $a^{p} \in E_{p+1}^{p}$.

We now show $E_{p+1}^{p} \varsubsetneqq E$ for every odd prime $p$. Suppose $E=E_{p+1}^{p}$. Then if we call $e$ the $(p+1) \times(p+1)$ matrix with 1 as the $1 \times 1$ entry and $0^{\prime} s$ elsewhere, we have $e=a_{1}^{p}+\ldots+a_{k}^{p}$ where $k$ is an integer greater than 1 and each $a_{i}$ is a non-trivial $(p+1)$-potent. Note that the $a_{i}^{\prime \prime s}$ are not necessarily distinct. Since each $a_{i}^{p}$ is an idempotent, the trace of $a_{i}^{p}, \operatorname{tr}\left(a_{i}^{p}\right)$, is a positive integer. Note that if $k>1,1=\operatorname{tr}(e)=\operatorname{tr}\left(\alpha_{1}^{p}\right)+\ldots+\operatorname{tr}\left(a_{k}^{p}\right)>1$. Therefore $e=a^{p}$ where $a=\left(\alpha_{i j}\right)$ is a non-trivial $(p+1)$-potent. Multiplying by $a$ we have $e a=a$. After equating entries, we get $\alpha_{i j}=0$ for $i \geq 2$. Remembering that $a^{p}=e$ we can equate $1 \times 1$ entries to obtain $\alpha_{11}^{p}=1$. Now if $\alpha_{11}=1, e=a^{p}=a$. But this means $a^{2}=a$ which is impossible since $a$ is a $(p+1)$-potent. Therefore $\alpha_{11}^{p-1}+\ldots+\alpha_{11}+1=0$. This means $Q$ splits $x^{p-1}+\ldots+x+1$, a contradiction when $p \geq 3$. So $E_{p+1}^{p} \varsubsetneqq E$.

EXAMPLE 2. Let $R$ be a simple ring whose center is the field $F$. It is easy to show that $E_{4}^{3}=E$ whenever $F$ splits $x^{2}+x+1$ and 
char $E \neq 3$. For if there exists an $f \in F$ such that $f^{2}+f+1=0$, then we know that $f \neq 1$ since char $F \neq 3$. If $e$ is a non-trivial idempotent of $A$, then $(f e)^{4}=f^{4} e^{4}=f e$. Now if $(f e)^{2}=f e$, then $f^{2}-f=0$. Since $f \neq 1, f=0$ which implies $1=0$, a contradiction. Note that for any element $a$ such that $a^{4}=a$ and $a^{2} \neq a$ we know that $a^{3} \neq a$. So fe is a 4-potent. Therefore for any $e \in E, e=(f e)^{3} \in E_{4}^{3}$. So $E \subset E_{4}^{3}$.

\section{Another set of generators}

If $n>1$ is the smallest integer such that $a+a^{2}+\ldots+a^{n-1}=0$ for some $a \in R$, then we call $a$ a pseudo-n-potent. Observe that if $a$ is a pseudo-n-potent non-trivial provided $a^{n-1} \neq 1,0$. By $P_{n}^{n-1}$ we mean the additive group generated by the $(n-1)$ st powers of non-trivial pseudo$n$-potents. We do not have to go far to find a ring with $P_{n}^{n-1} \neq \not$ as the next example shows.

EXAMPLE 3. Let $R$ be the ring of $(n-1) \times(n-1)$ matrices over a field. Consider the matrix

$$
a=\left(\begin{array}{ccccc}
0 & 1 & 0 & \ldots & 0 \\
0 & 0 & 1 & \ldots & 0 \\
\vdots & & & & \vdots \\
0 & 0 & 0 & \ldots & 1 \\
0 & -1 & -1 & \ldots & -1
\end{array}\right)_{(n-1) \times(n-1)}
$$

which is the companion matrix to $f(x)=x+x^{2}+\ldots+x^{n-1}$. Since $f$ is the minimal polynomial for $a, a$ is a pseudo-n-potent. Clearly $a^{n-1} \neq 1$ since $a$ has a column of zeros; and as before $a^{n-1}=0$ implies $a=0$ which is impossible if $n>2$.

THEOREM 2. Let $R$ be a ring having a non-trivial pseudo-n-potent. Then $P_{n}^{n-1}$ is a Lie ideal of $R$.

Proof. Let $a$ be a non-trivial pseudo-n-potent in $R$. From Lemma 1 
we learned that

$\left(a+a^{n-1} r-a^{n-1} r a^{n-1}\right)^{k}=a^{k-1}\left(a+a^{n-1} r-a^{n-1} r a^{n-1}\right)=a^{k}+a^{n+k-2} r-a^{n+k-2} r a^{n-1}$

whenever $a^{n}=a$. If we sum over $k$ from 1 to $n-1$, we have

$$
\begin{aligned}
\left(a+a^{n-1} r-a^{n-1} r a^{n-1}\right) & +\left(a+a^{n-1} r_{-a}^{n-1} r a^{n-1}\right)^{2}+\ldots+\left(a+a^{n-1} r-a^{n-1} r a^{n-1}\right)^{n-1} \\
& =\left(a+a^{2}+\ldots+a^{n-1}\right)+\left(a^{n-1}+a+a^{2}+\ldots+a^{n-2}\right) r \\
& -\left(a^{n-1}+a+a^{2}+\ldots+a^{n-2}\right) r a^{n-1} \\
& =0+0-0=0 .
\end{aligned}
$$

To show $n$ is minimal we suppose there exists a positive integer $t<n-1$ such that

$$
\begin{aligned}
0= & \left(a+a^{n-1} r-a^{n-1} r a^{n-1}\right)+\left(a+a^{n-1} r-a^{n-1} r a^{n-1}\right)^{2}+\ldots+\left(a+a^{n-1} r-a^{n-1} r a^{n-1}\right)^{t} \\
=\left(a+a^{2}+\ldots+a^{t}\right)+\left(a^{n-1}+a+a^{2}+\ldots+a^{t-1}\right) r & -\left(a^{n-1}+a+a^{2}+\ldots+a^{t-1}\right)_{r a}^{n-1} .
\end{aligned}
$$

Multiplying from the right by the idempotent $a^{n-1}$, we have $0=\left(a+a^{2}+\ldots+a^{t}\right) a^{n-1}=a^{n}+a^{n+1}+\ldots+a^{t+n-1}=a+a^{2}+\ldots+a^{t}$ which is a contradiction. Therefore $a+a^{n-1} r-a^{n-1} r a^{n-1}$ is a pseudo$n$-potent for every $r \in R$. To show that $a+a^{n-1} r-a^{n-1} r a^{n-1}$ is nontrivial we can use the same argument used in Lemma 1 . This means that

$$
\left(a+a^{n-1} r-a^{n-1} r a^{n-1}\right)^{n-1}=a^{n-1}+a^{n-2} r-a^{n-2} r a^{n-1}
$$

is a generator of $P_{n}^{n-1}$. If we replace $r$ with ar, we find that $a^{n-1}+a^{n-1} r-a^{n-1} r a^{n-1}$ is a generator of $a^{n-1}$ for every $r \in R$. In a similar manner we can show that $a^{n-1}+r a^{n-1}-a^{n-1} r a^{n-1}$ is a generator of $P_{n}^{n-1}$ for every $r \in R$. Since $P_{n}^{n-1}$ is an additive group we know that

$$
r a^{n-1}-a^{n-1} r=\left(a^{n-1}+r a^{n-1}-a^{n-1} r a^{n-1}\right)-\left(a^{n-1}+a^{n-1} r-a^{n-1} r a^{n-1}\right) \in P_{n}^{n-1} .
$$

So $P_{n}^{n-1}$ is a Lie ideal of $R$. 
COROLLARY 2. Let $A$ be a simple algebra of characteristic not equal to 2 having a non-trivial pseudo-n-potent. Then the subring generated by its pseudo-n-potents is $A$.

Proof. Follow the same argument used in the proof of Corollary 1.2.

We close with a conjecture.

CONJECTURE. Let $A$ be a simple algebra with a rational center. Let $a \in A$ such that $a^{3} \neq 1,0$. Then either $a, a^{2}$ and $a^{3}$ are linearly independent over the center, or $A$ contains a non-trivial pseudo-4-potent.

The proof of this conjecture will depend on a number theoretic problem. For if $\beta_{1} a+\beta_{2} a^{2}+\beta_{3} a^{3}=0$ where the $\beta_{i}$ 's are rational numbers, we can assume the $B_{i}{ }^{\prime} s$ are integers after clearing the equation of denominators. If we multiply this equation by $a$ and $a^{2}$, we get a homogeneous system whose coefficient matrix has determinant $\beta_{1}^{2}+\beta_{2}^{2}+\beta_{3}^{2}-3 \beta_{1} \beta_{2} \beta_{3}$ which must be zero. If in turn this implies $\beta_{1}=\beta_{2}=\beta_{3}$, we would know that $A$ contains a non-trivial pseudo- $n-$ potent.

\section{Reference}

[1] I.N. Herstein, Topics in ring theory (University of Chicago Press, Chicago and London, 1965).

Department of Mathematics,

University of Southwestern Louisiana,

Lafeyette,

Louisiana 70504,

USA. 